\title{
An Observation Model for Motion Correction in Nuclear Medicine
}

\author{
Majdi R. Alnowami ${ }^{a}$, E. Lewis ${ }^{a}$, M. Guy ${ }^{b}$ and K. Wells ${ }^{a}$ \\ ${ }^{a}$ Centre for Vision, Speech and Signal Processing, University of Surrey, Guildford,GU2 7XH; \\ ${ }^{b}$ Medway Maritime Hospital, Windmill Road, Gillingham, Kent, ME7 5NY, UK.
}

\begin{abstract}
This paper describes a method of using a tracking system to track the upper part of the anterior surface during scanning for developing patient-specific models of respiration. In the experimental analysis, the natural variation in the anterior surface during breathing will be modeled to reveal the dominant pattern in the breathing cycle. The main target is to produce a patient-specific set of parameters that describes the configuration of the anterior surface for all respiration phases. These data then will be linked to internal organ motion to identify the effect of the morphology of each on motion using particle filter to account for previously unseen patterns of motion. In this initial study, a set of volunteers were imaged using the Codamotion infrared marker-based system. In the marker-based system, the temporal variation of the respiratory motion was studied. This showed that for the 12 volunteer cohort, the mean displacement of the thorax surface TS ( abdomen surface AS) region is $10.7 \pm 5.6$ $\mathrm{mm}(16.0 \pm 9.5 \mathrm{~mm})$. Finally, PCA was shown to capture the redundancy in the data set with the first principal component (PC) accounting for more than $96 \%$ of the overall variance in both AS and TS datasets . A fitting to the dominant modes of variation using a simple piecewise sinusoid has suggested a maximum error of about $1.1 \mathrm{~mm}$ across the complete cohort dataset.
\end{abstract}

Keywords: Respiratory motion correction, PCA, Patient-specific models

\section{INTRODUCTION}

One of the current major challenges in nuclear emission tomography is respiratory motion correction. Respiratory motion during the emission data acquisition process leads to blurred images, thus challenging diagnosis, planning and follow-up processes. There is significant clinical evidence on the effect that respiratory motion has on lesions localisation. ${ }^{1-4}$ Correspondingly, significant tumour motion and variation in lung volume has been reported in ${ }^{5,6}$ whereby images reconstructed without respiratory motion compensation were studied. A significant research focus is in developing new approaches to monitor and track respiratory motion during data acquisition ${ }^{7}$. This may involve techniques to correct motion during reconstruction e.g. Reyes et al. ${ }^{6}$ or using a marker-based tracking system to monitor the body motion during data acquisition, e.g. Nehmeh et al. ${ }^{3}$, Boutchko et al. ${ }^{8}$ and Wolthaus et al., ${ }^{9}$. However, much of the prior work assumes invariant cyclic respiratory motion, where as in reality this behaviour is actually more complex.

The most simple approach for motion correction in nuclear imaging is the implementation of respiratory gating (RG). Generally, in RG the normal breathing cycle is divided into typically eight different phases. Dynamic NM data is then sorted into these eight gates, resulting in eight image sets with reduced motion. In the simplest case, only a single phase or gate is stored, thus minimizing the motion artefact. However, this yields statistically noisy images, as the majority of the data are ignored. Alternatively, respiratory gated images reconstructed with a reconstruction algorithm including some form of simple registration may be individually such as use of auto-correlation. In order to divide the motion into gated phases, a number of external instruments have been used for tracking respiratory motion such as pneumatic devices ${ }^{10}$ and IR tracking of the vertical position by two reflective markers. ${ }^{3}$ Others researchers ${ }^{8,11,12}$ have used stereo camera tracking systems with markers in various configurations arranged on the subject's anterior surface. The main disadvantage of these gating methods is the SNR (signal-to-noise ratio) in the reconstructed image of each phase is much lower that the SNR of the

Further author information: (Send correspondence to Majdi R. Alnowami.)

Majdi R. Alnowami: E-mail: m.alnowami@surrey.ac.uk

Medical Imaging 2010: Image Processing, edited by Benoit M. Dawant, David R. Haynor,

Proc. of SPIE Vol. 7623, 76232F - @ 2010 SPIE · CCC code: 1605-7422/10/\$18 - doi: 10.1117/12.844376

Proc. of SPIE Vol. $762376232 \mathrm{~F}-1$ 
un-gated PET image set so that, the reduced SNR leads to significant information loss. Therefore, gating alone appears insufficient for motion correction in nuclear medicine imaging. The Respiratory Correlated Dynamic (RCD) method was proposed to overcome the standard RG disadvantages. ${ }^{3,5}$ One of the major disadvantages of this method is that less data is used in each reconstruction and therefore the image suffers from statistical noise. Perhaps more importantly this approach used only one marker over the anterior surface to describe the internal organs motion and that is not enough to describe different pattern of breathing. As different breathing pattern cause a different internal organs motion.

In contrast to those other approaches, we propose a data driven scheme ${ }^{13,14}$ to achieve motion correction in nuclear medicine using a particle filtering frame work ${ }^{15}$ that relaxes commonly used assumptions about respiratory motion. As part os this scheme, this paper describes a method of using a marker-less tracking system to track the upper part of the torso during scanning for developing patient-specific models of respiration. Moreover, Principal component analysis (PCA) is used to study the breathing morphology for individual volunteers to characterize breathing behaviour. The main goal of the work presented here is to eventually develop a method of marker-less tracking of the upper part of the torso during scanning, and to parametrise patient-specific patterns of respiration. In the experimental analysis described later, the natural variation in the anterior surface during breathing will be modeled to reveal the dominant pattern in the breathing cycle. The main target is to produce a patient-specific set of parameters that completely describes the configuration of the anterior surface for all respiration phases. These data then will be linked to internal organ motion to identify the effect of the morphology of each on motion using particle filter to account for previously unseen patterns of motion.

\section{METHODOLOGY AND RESULTS}

An initial pilot study was undertaken using a set of volunteers, imaged using the Codamotion infrared markerbased tracking systems. A marker-less system is highly attractive in terms patient throughput but will generate Gigabytes of data per second of capture, which is cumbersome, at least, at the initial development stage. By contrast, the Codamotion system only captures marker coordinates and returns their 3D position in space generating only a few $\mathrm{kB}$ per second. Therefore, the Codamotion system was used in the preliminary study to analyse natural temporal variations in anterior wall configuration during breathing, and to understand how to parametrise individual respiratory morphology.

\subsection{Temporal motion study}

The Codamotion CX1 unit measures the 3D locations of infra-red active markers in real-time. The infra-red system Resolution is about $3 \mathrm{mrad}$ (0.002 degree) at $3 \mathrm{~m}$ from the camera with a lateral resolution of $0.1 \mathrm{~mm}$ and horizontal and vertical resolution of $0.6 \mathrm{~mm}$. The viewing angle of one CX1 unit is approx $80^{\circ}$. The sampling rate of the system was user-selected to 10 frame/sec. The markers for this system were arranged on each volunteer's anterior surface in a four-by-four grid ( see Figure 1(a)). The tracking points were arranged as follows: near the chest wall at the level of the third and fifth costal cartilage, section 1 (S1) and section 2 (S2) respectively, targeting the lung-apposed rib cage; midway between the xyphoid process and the costal margin (S3), targeting the abdomen-apposed rib cage; at the level of the umbilicus (S4).

\subsubsection{Volunteers Preparation}

In this initial study a set of seven male and five female volunteers having a significant variation in body shape, with body mass indices (BMI) ranging between $27.8 \mathrm{~kg} / \mathrm{m}^{2}$ and $21.3 \mathrm{~kg} / \mathrm{m}^{2}$, were imaged using the Codamotion marker-based system. Volunteers were asked to maintain the same overall position during the acquisitions and to breathe quietly for 10s. No others particular instruction regarding the breathing pattern was given. All volunteers were imaged in the supine position. Sixteen infra-red LED's were placed over the anterior surface as described previously and illustrated in Figure 1(b). 


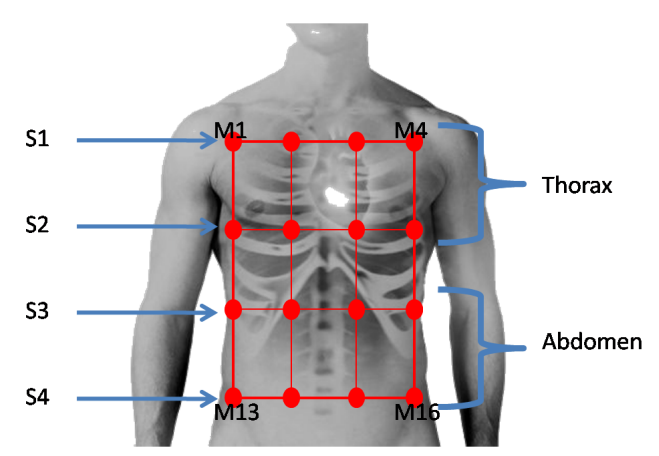

(a) The anterior grid consists of four horizontal rows and four equally spaced vertical column.

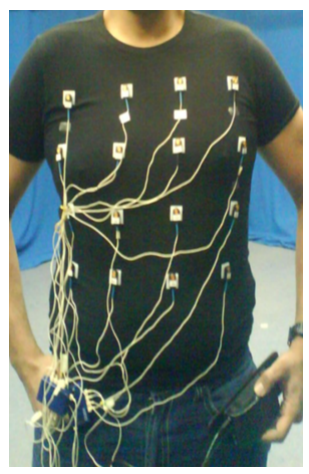

(b) Sixteen infra-red LED's were placed over the anterior surface of the volunteer

Figure 1. Tracking points arrangement on each volunteers anterior surface

\subsubsection{Data Acquisition}

As described in section 2.1, marker-based respiratory motion data were acquired using two $4^{*} 2$ groups of markers referred to as the Thoracic surface (TS) and the Abdomen surface (AS) respectively. Each frame was analysed as a column matrix $F_{n}$ :

$$
F_{n}=\left[X_{n, 1}, Y_{n, 1}, Z_{n, 1}, X_{n, 2}, Y_{n, 2}, Z_{n, 2} \ldots \ldots \ldots \ldots \ldots, X_{n, s}, Y_{n, s}, Z_{n, s}\right]^{T}
$$

where $X_{n, 1}, Y_{n, 1}, Z_{n, 1} \ldots . X_{n, s}, Y_{n, s}, Z_{n, s}$ describe the Cartesian data of each marker and 1 through $s$ is the marker index at frame $n$. As a result each frame will be represented by $D * 1$ vectors, where $D=3 * s$ is the dimensional vectors for $F_{n}$. Therefore, by using 8 markers for the TS and AS respectively, the representation of each data set is a 45 dimensional vector. The respiratory motion sequence for each volunteer is represented by a matrix $M$, where each row of the motion matrix corresponds to all the motion components of a particular marker, and each column of $M$ corresponds to a set of measurements from one particular frame. Equation 2 illustrates the motion matrix of the anterior surface.

$$
M=\left[F_{1}, F_{2}, F_{3}, \ldots \ldots \ldots \ldots \ldots \ldots, F_{99}, F_{100}\right], n=1: 100, \text { number } \text { of frames }
$$

\subsubsection{Results}

Respiratory motion can be defined by amplitude and period, where amplitude is defined by some measure of the displacement between maximum inhalation and maximum exhalation, and frequency is the number of breathing cycles per unit of time determined, for example, from baseline zero-crossing points or distance between peak inhalation. In these preliminary analyses, respiratory motion was studied as follows: the motion amplitude was used to understand and evaluate the maximum and minimum displacement in each marker under the hypothesis that the motion of the anterior surface is primarily defined by two factors TS and AS motion; ${ }^{16-18}$ secondly, the contribution of anterior-posterior (AP) (Z direction), lateral (L) (X direction), and superior-inferior (SI) (Y direction) motion to the total displacement of each marker was illustrated and analysed using PCA. Table 1 illustrates the results obtained from scanning the aforementioned volunteer cohort. It shows that the surface displacements for both TS and AS during a breathing cycle where these displacement values describe the average distance between the markers of a target (TS or AS) region. This table demonstrates a mean displacement of the TS (AS) region of $10.7 \pm 5.6 \mathrm{~mm}(16.0 \pm 9.5 \mathrm{~mm})$. In addition, the table indicates that the maximum displacement of TS is about $24.6 \mathrm{~mm}$ and the maximum displacement of AS is about $39.8 \mathrm{~mm}$.

To investigate the aforementioned hypothesis that anterior surface motion is controlled by the two components of TS and AS motion, the displacement of each marker from its mean position was plotted as a function of time. Figure 3 and 2 show an example of two different volunteers where M1 to M4 (solid lines) describe the TS variation, and M13 and M16 (dashed lines) describe the variation of AS where, M1 to M16 is marker(1) to marker(16) as 
described in Figure 1(a). In Figure 3 it can be seen that the displacement of AS and TS markers are in phase, and Figure 2 illustrates markedly different breathing patterns, where the AS and TS displacement are out of phase. These results are illustrative of the different respiratory motion patterns seen across the cohort. The amplitude of TS or AS displacement can also vary during the scanning time for the same individual.

\begin{tabular}{|l|l|l|l|l|}
\hline $\begin{array}{l}\text { Volunteer } \\
\text { No. }\end{array}$ & Gender & $\begin{array}{l}\text { BMI } \\
\left(\mathrm{kg} / \mathrm{m}^{2}\right)\end{array}$ & $\begin{array}{l}\text { TS Displace- } \\
\operatorname{ment}(\mathrm{mm})\end{array}$ & $\begin{array}{l}\text { AS Displace- } \\
\operatorname{ment}(\mathrm{mm})\end{array}$ \\
\hline 1 & M & 28.2 & 12.5 & 39.8 \\
\hline 2 & M & 24.3 & 11.3 & 27.9 \\
\hline 3 & M & 25.2 & 24.6 & 8.5 \\
\hline 6 & M & 21.8 & 18.4 & 11.0 \\
\hline 7 & M & 27.8 & 6.8 & 12.7 \\
\hline 4 & M & 24.8 & 10.3 & 10.8 \\
\hline 9 & M & 21.4 & 13.6 & 25.7 \\
\hline 5 & F & 25.2 & 13.5 & 9.6 \\
\hline 8 & F & 24.6 & 7.4 & 15.6 \\
\hline 10 & F & 22.2 & 7.6 & 22.7 \\
\hline 11 & F & 25.5 & 7.1 & 20.3 \\
\hline 12 & F & 21.7 & 5.1 & 24.6 \\
\hline \multicolumn{2}{|c|}{ Cohort average } & 23.6 & 10.7 & 16.0 \\
\hline Standard Deviation & 2.3 & 5.6 & 9.5 \\
\hline \multicolumn{2}{|c|}{ Max } & 28.2 & 24.6 & 39.8 \\
\hline \multicolumn{2}{|c|}{ Min } & 21.4 & 5.1 & 8.5 \\
\hline
\end{tabular}

Table 1. Mean experimental surface displacements for both chest and abdominal region during a 10s breathing cycle acquisition for a group of 12 male and female volunteers.

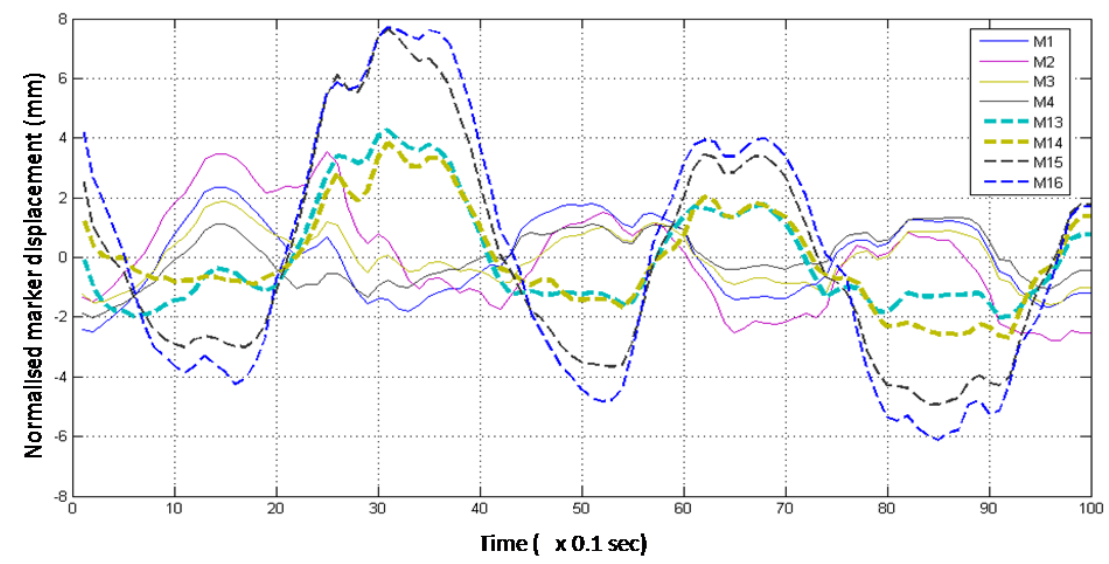

Figure 2. The volunteer breathing pattern shows the displacement of AS and TS markers are out of phase with a noticeable change in amplitude and frequency

To understand the dominant motion component, the motion of each marker was analysed. Figure 4(a) illustrates the motion path for markers of one volunteer in Cartesian space. A straight line was then fitted through each marker as illustrated in Figure 4(b).

Figure 5 illustrates the contribution of each displacement direction to the total amplitude of the displacement during the breathing cycle for the 12 volunteers, calculated using:

$$
\text { Contribution }=\frac{C^{2}}{S I^{2}+A B^{2}+L^{2}}
$$




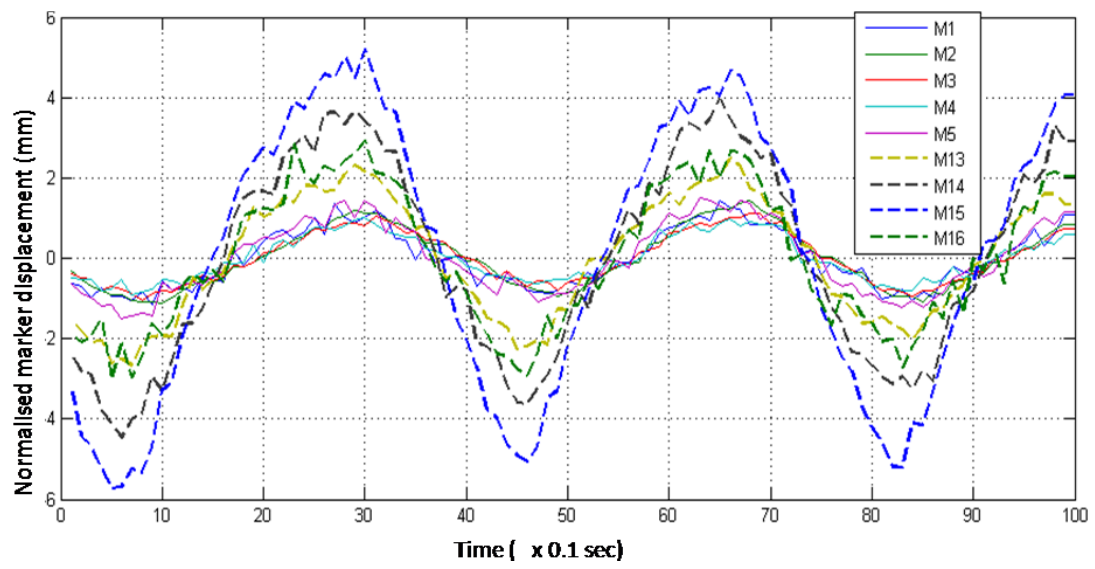

Figure 3. This Figure shows that the displacement of AS and TS markers are in phase and there is amplitude and frequancy of the breathing cycle almost constant

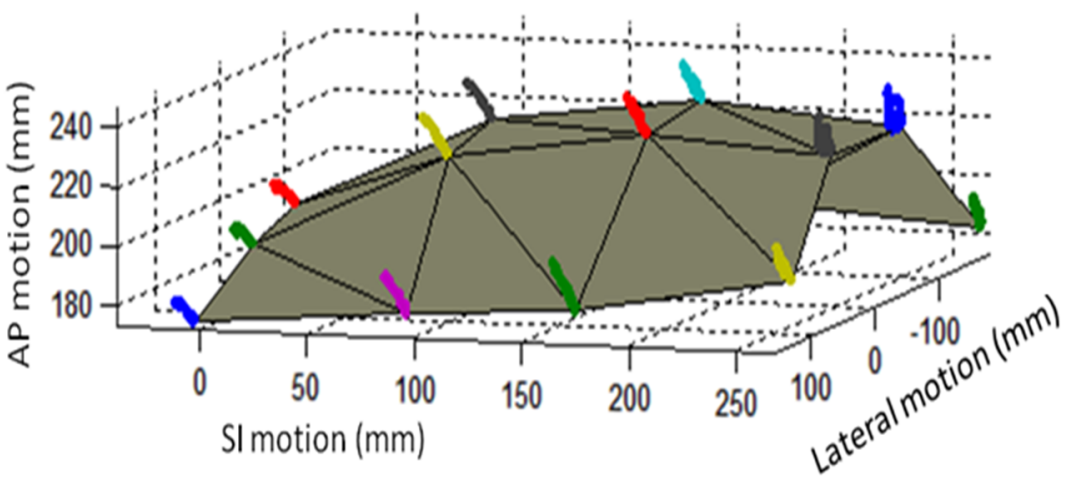

(a) Surface representation of the anterior surface, showing marker displacement. This illustrates the motion path for all the markers of one volunteer in $3 \mathrm{D}$.

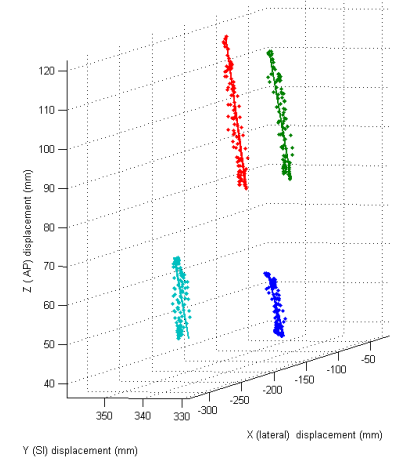

(b) Illustrates a straight line fitting through each marker motion path.

Figure 4. The Cartesian displacement of each marker during the respiratory cycle.

where $\mathrm{C}$ is the Cartesian displacement in SI, $\mathrm{AB}$ or $\mathrm{L}$ direction. This figure illustrates that the contribution of AP motion is about $95 \% \pm 3 \%$ of the total amplitude of the higher breathing pattern (amplitude in the range 27 to $40 \mathrm{~mm}$ ), falling to less than $40 \% \pm 10 \%$ of the tidal breathing pattern (amplitude in the range 7 to 12 $\mathrm{mm}$ ). Conversely, the contribution of SI and L motion is less than $5 \% \pm 2 \%$ of the total amplitude of the largest amplitude, rising to about $50 \% \pm 8 \%$ of the tidal data. Therefore, in deep breathing, the contribution of SI and L motion might be neglected.

\section{ANALYSIS}

The evidence of dominant behavior shown in the previous section suggests that PCA analysis could successfully be used to effectively represent the anterior surface motion. To start this process, the representation of each data set is assigned to a 24-dimensional vector. Each frame within the respiratory cycle $F(n)$ where $n(=1, \cdots, 100)$ is a time index, was described as combination of $F_{A S}(n)$ and $F_{T S}(n)$ i.e. the thoracic and abdomen marker sets. PCA analysis was then undertaken. The first seven principal components for each data set are illustrated in Figure 6. On average, the first principal component (PC) accounts for more than $96 \%$ of the overall variance in both data sets AS and TS. Obviously, PCA is very successful in capturing the redundancy in the data.

Figure 7 illustrates the dominant modes of variation associated with the dominant eigenspace for one of the volunteers, where $W_{T S}$ and $W_{A S}$ describes the dominant modes of variation for the TS and AS motion 


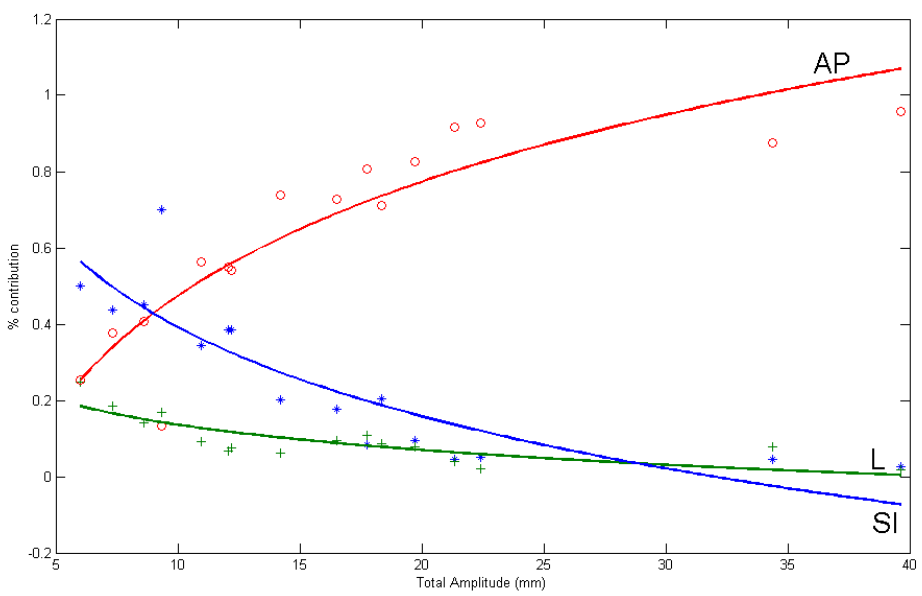

Figure 5. Illustrates the contribution of each Cartesian displacement to the total amplitude of the displacement during the breathing cycle. The solid line shows a logarithmic fitting for the average contribution of the Cartesian displacement $\mathrm{SI}$ (blue) , $\mathrm{AB}$ (red) and L(green) at different amplitude.

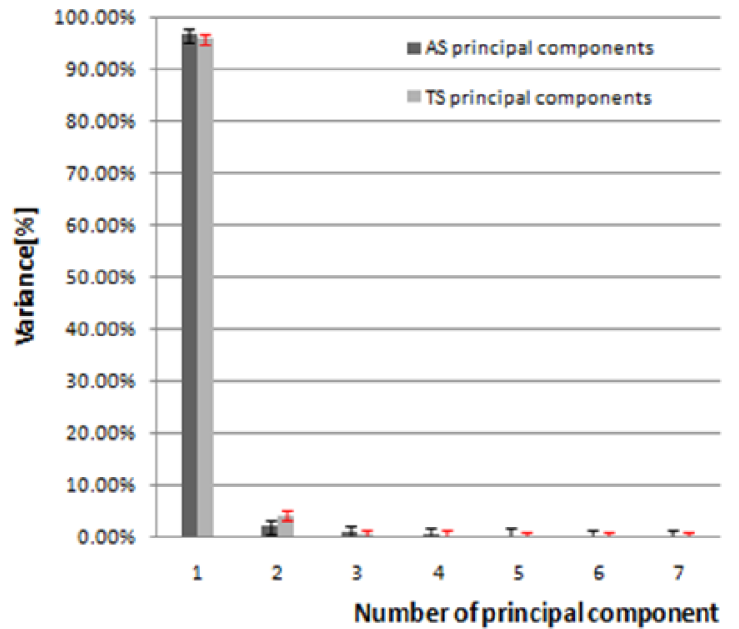

Figure 6. The first seven principal components. The error bars represent the standard deviation on the calculated values across the cohort

respectively where $W_{T S}(n)$ and $W_{A S}(n)$ represents the weight of the first eigenvectors $e_{A S}(1)\left(e_{T S}(1)\right)$ for each frame $F_{n}$ for $n=1: 100$ Therefore, each frame within the respiratory motion can be described by equation 4 :

$$
F(n)= \begin{cases}F_{T S}(n) & : \text { for } T S \\ F_{A S}(n) & : \text { for } A S\end{cases}
$$

where $F_{A S}(n)$ and $F_{T S}(n)$ are the vector data for AS and TS at frames $n$. These are given by a linear combination of the mean vectors $F_{A S}(0)$ and $F_{T S}(0)$ respectively plus the weighted first eigenvectors $e_{A S}(1)\left(e_{T S}(1)\right)$ for each surface as given in equation 5 :

$$
F_{A S}(n)=e_{A S}(1) * W_{A S}(n)+F_{T S}(0), F_{T S}(n)=e_{T S}(1) * W_{T S}(n)+F_{T S}(0)
$$

where $W_{A S}(n)\left(W_{T S}(n)\right)$ the weight/score of $e_{A S}(1)\left(e_{T S}(1)\right)$ at each frame $n$. Since the mode of variation $W$ is a cyclic motion over time. Therefore, each cycle within the motion sequence might be approximated in pseudo sinusoidal form similar to the parameterization given in. ${ }^{19,20}$ A preliminary piecewise sinusoidal fitting 


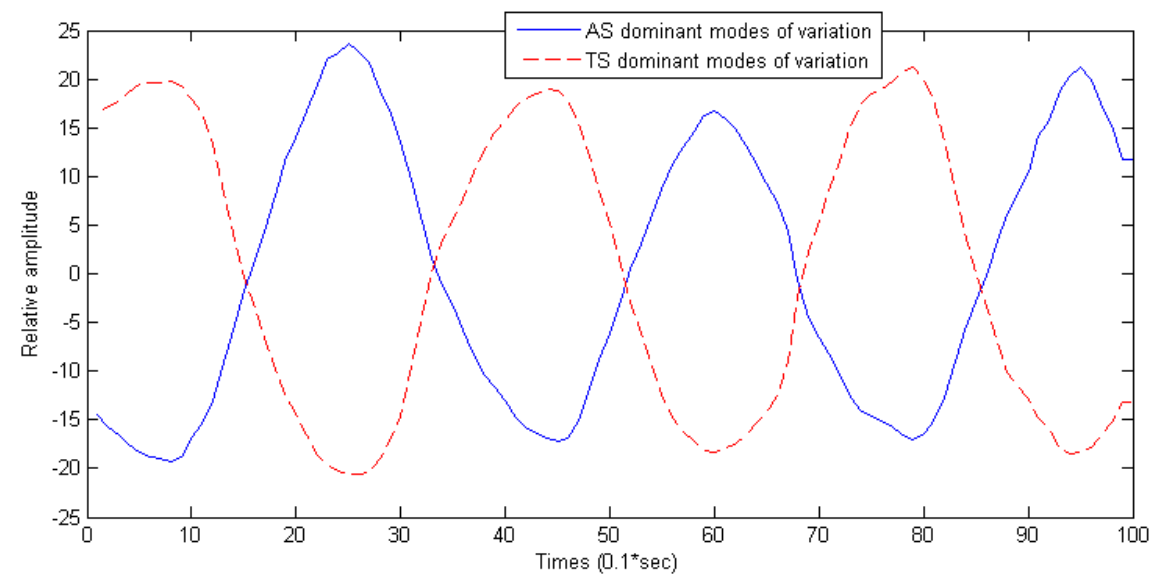

Figure 7. Illustrates the dominant modes of variation for $W_{T S}$ and $W_{A S}$ for the TS and AS respectively following PCA analysis.

is illustrated in Figure 8. The error associated with this fitting is also illustrated, with maximum error of about $1.1 \mathrm{~mm}$ for this initial case. Table 2 illustrates sinusoidal fitting parameters for each half cycle and the maximum error presented in each fitting.

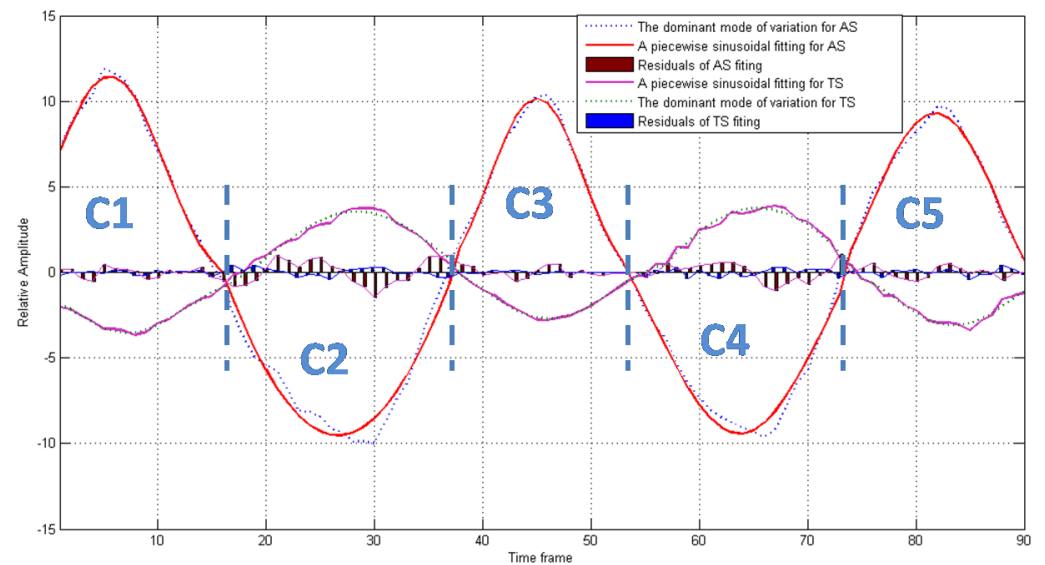

Figure 8. Illustration of the dominant modes of variation (the coefficient of the first eigenvectors) changing over time for 90 frames. It also illustrates the piecewise sinusoidal fitting for each half cycle $\mathrm{C} 1, . ., \mathrm{C} 5$. The bars illustrates the residual error associated with each fitting

As a result, the phase of the interior surface at any particular time during the respiratory cycle can be parametrized using these sine equation thus to describe the breathing pattern of an individual, there are five components required the average frame $F(0)$, the first eigenvectors $e(1)$, the fundamental frequency $f$, amplitude amp and the phase $\phi$.

\section{DISCUSSION AND CONCLUSION}

In this initial study, a set of volunteers were imaged using the Codamotion infrared marker-based system. In the marker-based system, the temporal variation of the respiratory motion was studied. This showed that for the 12 volunteer cohort, the mean displacement of the TS (AS) region is $10.7 \pm 5.6 \mathrm{~mm}(16.0 \pm 9.5 \mathrm{~mm})$. We 


\begin{tabular}{|l|l|l|l|l|l|}
\hline Half Cycle & Surface & $\begin{array}{l}\text { Frequency } \\
(\mathrm{HZ})\end{array}$ & $\begin{array}{l}\text { Amplitude } \\
(\mathrm{mm})\end{array}$ & $\begin{array}{l}\text { Phase (radi- } \\
\text { ans })\end{array}$ & $\begin{array}{l}\text { Max error } \\
(\mathrm{mm})\end{array}$ \\
\hline \multirow{2}{*}{ C1 } & TS & 0.53 & 1.18 & -3.6 & 0.21 \\
\cline { 2 - 6 } & AS & 0.47 & 11.4 & 0.21 & 0.53 \\
\hline \multirow{2}{*}{ C2 } & TS & 0.31 & 2.51 & -1.05 & 0.42 \\
\cline { 2 - 6 } & AS & 0.19 & -9.5 & 0.72 & 1.44 \\
\hline \multirow{2}{*}{ C3 } & TS & 0.41 & -1.67 & -0.57 & 0.34 \\
\cline { 2 - 6 } & AS & 0.56 & 19.1 & -0.88 & 0.52 \\
\hline \multirow{2}{*}{ C4 } & TS & 0.14 & 13.06 & 0.84 & 0.23 \\
\cline { 2 - 6 } & AS & 0.314 & -9.4 & -0.59 & 1.1 \\
\hline \multirow{2}{*}{ C5 } & TS & 0.44 & -1.66 & -0.52 & 0.42 \\
\cline { 2 - 6 } & AS & 0.41 & 9.3 & -0.34 & 0.54 \\
\hline
\end{tabular}

Table 2. Illustrates sinusoidal fitting parameters for each half cycle and the maximum error presented in each fitting

might assume that this is a reasonable approximation to patient behavior. Variation between the TS and AS displacement in the same individual has also been observed, which indicates that the temporal displacement in $\mathrm{AS}$ and TS is inconsistent demonstrating that thoracic/abdomen dominant breathing may occur/change during respiratory motion. Furthermore, the study has show that the contribution of AP motion is about $95 \% \pm 3 \%$ of the total amplitude during deep breathing, falling to less than $40 \% \pm 10 \%$ during tidal breathing. Conversely, the contribution of SI and L motion is less than $5 \% \pm 2 \%$ of the total amplitude of the higher breathing pattern, rising to about $50 \% \pm 8 \%$ of the tidal breathing pattern. Finally, PCA was shown to capture the redundancy in the data set with the first principal component (PC) accounting for more than $96 \%$ of the overall variance in both AS and TS datasets. A fitting to the dominants modes of variation using a simple piecewise sinusoid has suggested a maximum error of about $1.1 \mathrm{~mm}$ across the complete cohort dataset. This will be used to parametrize respiratory motion, and feed into our particle filtering based approach at motion correction in nuclear medicine.

\section{ACKNOWLEDGMENTS}

We would like to acknowledge the assistance of Prof. Adrine hilton Visiual Media group, University of Surrey in providing access to the camera tracking system used in this work. Moreover, MRA would like to acknowledge financial support from King Abdulaziz University.

\section{REFERENCES}

[1] Knopp, M. V. and Bischoff, H. G., "Evaluation of pulmonary lesions with positron emission tomography," Radiologe 34(10), 588-91 (1994).

[2] Nehmeh, S. A., Erdi, Y. E., Ling, C. C., Rosenzweig, K. E., Schoder, H., Larson, S. M., Macapinlac, H. A., Squire, O. D., and Humm, J. L., "Effect of respiratory gating on quantifying pet images of lung cancer," $J$ Nucl Med 43(7), 876-81 (2002).

[3] Nehmeh, S. A., Erdi, Y. E., Rosenzweig, K. E., Schoder, H., Larson, S. M., Squire, O. D., and Humm, J. L., "Reduction of respiratory motion artifacts in pet imaging of lung cancer by respiratory correlated dynamic pet: methodology and comparison with respiratory gated pet," J Nucl Med 44(10), 1644-8 (2003).

[4] Daouk, J., Fin, L., Bailly, P., and Meyer, M. E., "Respiratory-gated positron emission tomography and breath-hold computed tomography coupling to reduce the influence of respiratory motion: methodology and feasibility," Acta Radiol 50(2), 144-55 (2009).

[5] Nehmeh, S. A., Erdi, Y. E., Pan, T., Yorke, E., Mageras, G. S., Rosenzweig, K. E., Schoder, H., Mostafavi, H., Squire, O., Pevsner, A., Larson, S. M., and Humm, J. L., "Quantitation of respiratory motion during 4d-pet/ct acquisition," Med Phys 31(6), 1333-8 (2004).

[6] Reyes, M., Malandain, G., Koulibaly, P. M., Gonzalez-Ballester, M. A., and Darcourt, J., "Model-based respiratory motion compensation for emission tomography image reconstruction," Physics in Medicine and Biology 52(12), 3579-3600 (2007).

[7] Rahmim, A., Rousset, O., and Zaidi, H., "Strategies for motion tracking and correction in pet," 2, 251-266 (Apr. 2007). 
[8] Boutchko, R., Balakrishnan, K., Reutter, B. W., and Gullberg, G. T., "Patient motion correction in computed tomography by reconstruction on a moving grid," NSS '0\%. IEEE 4, 2705-2707 (2007).

[9] Wolthaus, J. W. H., Herk, M. V., Muller, S. H., Belderbos, J. S. A., Lebesque, J. V., Bois, J. A. D., Rossi, M. M. G., and Damen, E. M. F., "Fusion of respiration-correlated pet and ct scans: correlated lung tumour motion in anatomical and functional scans," Physics in Medicine and Biology 50, 1569-1583 (April 2005).

[10] Lang, N., Dawood, M., Bther, F., Schober, O., Schfers, M., and Schfers, K., "Organ movement reduction in pet/ct using dual-gated list-mode acquisition," Zeitschrift fr medizinische Physik 16(1), 93-100 (2006).

[11] Beach, R. D., Pretorius, P. H., Boening, G., Bruyant, P. P., Feng, B., Fulton, R. R., Gennert, M. A., Nadella, S., and King, M. A., "Feasibility of stereo-infrared tracking to monitor patient motion during cardiac spect imaging," IEEE Trans Nucl Sci 51(5 II), 2693-2698 (2004).

[12] Bruyant, P. P., Gennert, M. A., Speckert, G. C., Beach, R. D., Morgenstern, J. D., Kumar, N., Nadella, S., and King, M. A., "A robust visual tracking system for patient motion detection in spect: Hardware solutions," IEEE Trans Nucl Sci 52(5 I), 1288-1294 (2005).

[13] Wells, K., Goswami, B., Rahni, A., Jones, J., and M. Alnowami, E. L., "A flexible approach to motion correction in nuclear medicine," IEEE Medical Imaging Conf, Orlando, Florida, USA (2009).

[14] Jones, J. and et al., K. W., "A virtual dissection based registration to model patient-specific respiratory motion," IEE, Orlando, Florida, USA (2009).

[15] Rahni, A., Lewis, E., Guy, M., and Wells, K., "Development of a particle filter framework for respiratory motion correction in nuclear medicine imaging," SPIE, San Diego, California, USA (2010).

[16] Konno, K. and Mead, J., "Measurement of the separate volume changes of rib cage and abdomen during breathing," J Appl Physiol 22(3), 407-22 (1967).

[17] Ferrigno, G., Carnevali, P., Aliverti, A., Molteni, F., Beulcke, G., and Pedotti, A., "Three-dimensional optical analysis of chest wall motion," J Appl Physiol 77(3), 1224-31 (1994).

[18] De Groote, A., Wantier, M., Cheron, G., Estenne, M., and Paiva, M., "Chest wall motion during tidal breathing," J Appl Physiol 83(5), 1531-7 (1997).

[19] Okwechime, D. and Bowden, R., "A generative model for motion synthesis and blending using probability density estimation," AMDO , 218-227, Springer-Verlag, Berlin, Heidelberg (2008).

[20] Troje, N. F., "Decomposing biological motion: A framework for analysis and synthesis of human gait patterns," J. Vis. 2, 371-387 (9 2002). 